\title{
Dalla periferia al centro: un secolo di storie di irpini emigrati in Nord America (1911-2010)*
}

\section{From the Periphery to the Centre: 100 Years of Stories \\ of Irpinians Emigrated in North America (1911-2010)}

Francesco Bianco [francesco.bianco@upol.cz]

Univerzita Palackého v Olomouci, Repubblica Ceca

\section{RiAsSUNTO}

Lobiettivo di questo contributo è presentare i temi principali di una raccolta di lettere scritte, nell'arco di un secolo, da italiani (originari dell'Irpinia) emigrati in Canada e negli Stati Uniti. Salute, morte, tappe della vita, famiglia, religione, denaro, vita contadina e urbana, viaggi sono le tematiche più ricorrenti in testi che, oltre a colpire per una talora spiccata vena narrativa, gettano una luce sulla società italoamericana dell'ultimo secolo. Il lavoro si inscrive nel quadro del progetto di ricerca Jazyk italský emigrantů (la lingua degli emigranti italiani: www.francescobianco.net/linguistica/progetti/emigrazione).

\section{Parole chiave}

America; Canada; Emigrazione; Irpinia; Italia; Lettere; Lingua; Stati Uniti

\begin{abstract}
The aim of this work is to present the main topics of a collection of letters written during 100 years by Italians (from Irpinia) who had migrated to Canada and to the U.S. Health, death, steps of life, family, religion, money, life in the countryside vs life in the city, journeys, are the most common topics in these texts. They sometimes strike the reader, due to their strong narrative vain, and they shed light on Italoamerican society of the last century. The work has been developed within the framework of the research project Jazyk italský emigrantů (the language of Italian emigrants: www.francescobianco.net/linguistica/progetti/ emigrazione).
\end{abstract}

\section{KEYWORDS}

America; Canada; Emigration; Irpinia; Italy; Letters; Language; United States

RICEVUTO 2015-08-31; ACCETTATO 2015-12-21

\footnotetext{
* Il presente contributo è parte integrante del progetto Jazyk italských emigrantů (La lingua degli emigranti italiani; http://www.francescobianco.net/linguistica/progetti/emigrazione/) finanziato dal Fond pro podporu vědecké činnosti (2014-2016; numero del progetto: 452100461). Nel 2016 il finanziamento è stato prorogato di un altro anno (fino al 2017). I fini del progetto sono spiegati a p. 134, nota 2.
} 
Il fenomeno dell'emigrazione italiana nel mondo, in particolare quello dell'emigrazione postunitaria, è stato oggetto di numerose indagini, che hanno coinvolto e continuano a coinvolgere studiosi di diversa estrazione.

Negli ultimi anni si è registrata una crescente mole di iniziative dedicate interamente o parzialmente a questo tema: mostre, convegni, pubblicazioni e borse di studio. Basterà citare, a titolo d'esempio, due opere diverse ma entrambe fondamentali, uscite a dieci anni di distanza l'una dall'altra: i due volumi di Storia dell'emigrazione italiana di Bevilacqua, De Clementi, \& Franzina (2001-2002) e la più recente Storia linguistica dellemigrazione italiana nel mondo di Vedovelli (2011) ${ }^{1}$.

A questo filone di studi va ascritto anche il progetto Jazyk italský emigrantů (la lingua degli emigranti italiani), ideato da chi scrive e attualmente finanziato dall'Università Palacký di Olomouc $^{2}$. Il fine del progetto è duplice: pubblicare una raccolta di lettere scritte da italiani emigrati all'estero e studiarne a fondo i caratteri linguistici, affiancando analoghi studi già esistenti sull'argomento. L'esistenza di questa raccolta si deve all'iniziativa di Salvatore Boniello, maestro elementare nato e vissuto a Guardia Lombardi (AV), con una lunga esperienza nel campo della promozione linguistica e culturale locale ${ }^{3}$.

Fra le numerose ricerche condotte da Boniello, una riguardava i compaesani emigrati all'estero: per decenni egli raccolse le lettere scritte da questi migranti e le archiviò; l'intenzione dello studioso, probabilmente, era quella di pubblicare, prima o poi, il proprio epistolario personale (Boniello, infatti, è uno dei destinatari di questi documenti), arricchito nel tempo con lettere provenienti da altri archivi famigliari, appartenenti a persone a lui evidentemente vicine. La morte, sopraggiunta nel 2010, impedì a Boniello di portare a termine il suo progetto; progetto successivamente ripreso, in sede accademica, con taglio e con scopi sensibilmente diversi, dal sottoscritto. La raccolta, visionata e scansionata interamente nell'estate del 2014, è formata da 272 fra lettere e biglietti (per lo più natalizi), più alcuni documenti che, con ogni probabilità, accompagnarono le lettere (e i biglietti) nei loro viaggi verso l'Italia: fotografie ${ }^{4}$, fogli di accompagnamento di merci, etichette e buste, alcune delle quali sopravvissute al loro contenuto. L'archivio, purtroppo, contiene solo documenti spediti dall'estero all'Italia ${ }^{5}$; Boniello non fu in grado, ammesso che vi abbia mai provato, di recuperare le missive che viaggiarono in direzione opposta (dall'Irpinia alle comunità emigrate). L'arco cronologico nel

1 Per quel che riguarda lo specifico dell'emigrazione italiana negli Stati Uniti, merita almeno un cenno il poderoso sforzo di LaGumina et al. (2000).

2 Il progetto (http://www.francescobianco.net/linguistica/progetti/emigrazione/) ha ricevuto un finanziamento nel 2014, nell'ambito del Fond pro podporu vědecké činnosti. È stato presentato, nel novembre dello stesso anno, in occasione del $17^{\circ}$ incontro dei romanisti cechi “Česká romanistika v evropském kontextu”, svoltosi a Olomouc (alcune considerazioni fatte in tale occasione confluiscono nel presente contributo). Un primo sondaggio, su pochi testi della raccolta, era stato già compiuto qualche anno prima (cfr. Bianco 2013).

3 Per un breve profilo biografico di Salvatore Boniello mi permetto di rinviare a Bianco (2013: 102-103, nota 3).

4 Come sottolineano Gibelli \& Caffarena (2001: 573), le "fotografie [...] formano insieme alle lettere un funzionale insieme integrato di comunicazione" famigliare.

5 Diverse furono le destinazioni degli emigranti guardiesi: le vie più battute sono quelle li condussero nel continente americano, in particolare in Argentina, negli USA (soprattutto in Pennsylvania; cfr. Longo 2004) e in Canada. Nel presente studio si considerano soltanto lettere di persone emigrate in nord America (quasi tutte negli Stati Uniti). Il rapporto fra campani emigrati in Canada e le loro comunità di origine è trattato in De Biase (2012). 
quale sono compresi i documenti è di circa cento anni: dagli anni Dieci del XX fino al primo decennio del XXI secolo.

Fin dal loro primo e parziale esame, i testi hanno esibito caratteri linguistici interessanti, sebbene non sorprendenti rispetto a documenti simili, analizzati da altri studiosi. Riassumendo quanto osservato già in Bianco (2013) e integrando i dati ivi presentati con quelli tratti dallo spoglio di altri documenti della raccolta, passo a elencare, esemplificandoli, i principali tratti salienti delle lettere.

Nell'ambito della grafia e della mise en page, si osserva, in generale, il mancato rispetto di molte convenzioni dell'italiano e della scrittura epistolare in genere: disordinata disposizione o assenza degli elementi paratestuali (data, luogo, firma); fenomeni di errata segmentazione (agglutinazione: 1a; deglutinazione: 1b); carenza (1c) o, più raramente, sovrabbondanza (1d) di segni di interpunzione e paragrafematici; assenza dell' $h$ diacritica nelle forme di avere (1e):

1) [a] lamerica 'l'America', anessuno 'a nessuno', unaltro 'un altro' [b] al quando 'alquanto', lo perazione 'loperazione' ${ }^{\prime}[\mathbf{c}]$ scrivero 'scriverò', proprieta 'proprietà' [d] in'America 'in America' $[\mathbf{e}] o$, anno 'ho, hanno"

A livello fonetico e fonologico, i testi esibiscono tratti tipici delle parlate (dialetti e italiano regionale) meridionali intermedie, soprattutto a livello di consonantismo: assimilazione progressiva del nesso [nd] (2a); sonorizzazione dell'occlusiva postnasale $(2 \mathrm{~b})^{8}$; affricazione della sibilante postnasale (2c); desonorizzazione della consonante intervocalica (2d). Questi fenomeni, evidentemente marcati a livello sociolinguistico (cioè stigmatizzati dai parlanti più colti), producono anche interessanti reazioni ipercorrette $(2 \mathrm{e})^{9}$ :

2) [a] danno 'dando' [b] cambione 'campione', raccondato 'raccontato' [c] penzare 'pensare' [d] macari 'magari' [e] mutante 'mutande', fatiga 'fatica'

I fenomeni morfologici più diffusi sono i metaplasmi, di declinazione $(3 \mathrm{a})^{10}$ e di genere $(3 \mathrm{~b})$; non mancano deviazioni nei paradigmi della flessione verbale, sollecitate dall'analogia (3c) o da forme dialettali soggiacenti $(3 \mathrm{~d})^{11}$ :

3) [a] bicchiero 'bicchiere', moglia 'moglie', signoro '[il] signore' [b] migli 'miglia', odia '[l'] odio' [c] serva '[egli, ella] serve'12 [d] potemo 'possiamo'

6 Cfr. Bianco (2013: 105).

7 Per rispetto della veste linguistica dei testi si preferisce riprodurre vocaboli e brani delle lettere in trascrizione diplomatica, conservandone le deviazioni grafiche rispetto alla norma. La lineetta obliqua indica l'accapo.

8 Cfr. anche $1 \mathrm{~b}$.

9 Cfr. Bianco (2013: 106).

10 Cfr. anche saluta, in $4 \mathrm{~b}$, e commara, in $5 \mathrm{~b}$.

11 Cfr. Bianco (2013: 108).

12 Per analogia, forse, con la terza persona dell'indicativo presente della prima coniugazione. 
La sintassi, poco sostenuta dalla (scarsa) padronanza interpuntiva e morfologica, è prevalentemente paratattica. Alcuni fenomeni caratterizzanti riguardano l'ambito morfosintattico: in $4 \mathrm{a}$ è esemplificato il che polivalente; in $4 \mathrm{~b}$ si osserva una reggenza verbale non standard (godere + oggetto diretto). Sulla costruzione della frase possono influire modelli diversi; l'inglese può essere il modello in $4 \mathrm{c}$ (the same as $\rightarrow$ lo stesso come), mentre $4 \mathrm{~d}$ esibisce un aggettivo relativo ${ }^{13}$, costrutto diffuso nei testi amministrativi:

4) [a] mi dispiace che non può venire in'America che se no avrebbe stato un'iuto per voi [b] spero di voi tutti che godete una perfetta saluta $[\mathbf{c}]$ spero che lanno venturo scriverò lo stesso come quest'anno [d] Includo alla presenta trovate un piccolo vaglio di Lire 110 la quale somma sono intestate a nostra madre

La lingua della pubblica amministrazione agisce come modello anche sulle scelte lessicali, come mostrano i burocratismi riportati in $5 \mathrm{a}^{14}$; altre componenti del lessico di queste lettere sono i regionalismi (5b), i malapropismi (5c) e i forestierismi ${ }^{15}(5 \mathrm{~d})$ :

5) [a] detti 'già nominati in precedenza' (credo che sul ritardo dei detti oggetti non avrai penzato a nulla di male), presenta 'presente'16 ( $m$ i Auguro che non sarai sotto le armi al giungere della presenta [= la lettera che stai leggendo]) [b] $\operatorname{com}(m)$ ara 'madrina di battesimo o di cresima, testimone di nozze ${ }^{17}$, tenere 'avere, possedere' (solo contento che la casa la tiene [= possiede/ possieda] lei) [c] il dottore che lo esiste [in luogo di assiste] è un Italiano di Alesandria [d] bosso 'capo, padrone'18 ( $\leftarrow$ ingl. boss), ingi 'pollici [unità di misura]' ( $\leftarrow$ ingl. inch [es])

Detto dei caratteri linguistici, si passa a considerare, nelle pagine che seguono, i contenuti delle lettere: documenti, fra gli altri (diari, libri di memorie, romanzi), dell'emigrazione italiana, vista con gli occhi dei suoi protagonisti ${ }^{19}$. Gli argomenti trattati offrono uno spaccato storico, sociologico e psicologico del fenomeno migratorio italiano, trovando precise rispondenze nei contenuti di altri epistolari simili ma aggiungendo dettagli, forse non del tutto irrilevanti, su questioni riguardanti la vita di specifiche comunità.

Un primo tema, presente in quasi tutte le lettere e di solito concentrato nelle righe di apertura, riguarda la salute e la malattia, spesso conseguenza di una vita difficile, condotta in condizioni precarie, che non sempre l'emigrazione ha migliorato. La salute è oggetto di auguri ma anche di veri e propri resoconti (6d, 6e), spesso poco incoraggianti, e di richieste di informazioni (6b):

13 Su questo costrutto mi permetto di rinviare ad alcuni miei lavori precedenti: Bianco $(2004,2009,2012)$.

14 Sull'italiano burocratico come modello per gli scriventi semicolti cfr. D’Achille (1994: 73) e Bianco (2016).

15 La lingua straniera era "la grande assente" nei dati presentati in Bianco (2013: 111-112): in tale occasione si era supposto che questa assenza, del tutto casuale, fosse legata al numero ristretto di testi fino ad allora spogliati.

16 Cfr. Bianco (2013: 109; 2016: 521).

17 Nei dialetti irpini questo vocabolo può avere anche il significato di 'amante’ (cfr. Bianco 2013: 108); significato che il contesto di queste lettere consente di escludere sistematicamente.

18 Boss è una parola chiave dell'emigrazione italiana negli USA. Così erano chiamati i mediatori "fra gli immigrati disorientati e quel paese così estraneo" (Vecoli 2002: 58), cioè gli Stati Uniti. Dietro il pagamento di un onorario, chiamato bossatura, questi mediatori procuravano all'emigrante alloggio e lavoro.

19 Pochi cenni ai contenuti delle lettere sono già in Bianco (2013: 103-105). 
6) [a] Sono lieto nel sentire che tutti di nostra casa state bene [b] Cara commara fammi sapere voi come state credo che godete in buona salute assieme a tutti di famiglia [c] Da tanto tempo voleva scriverti ma sono stato ammalato e non ti ho scritto [d] la sorella da quando in quando soffre di malattie e romotesime nelle bracce e verso la fina di settembre ebbe unaltra paura brutta cioè mi cadde ammalata $X^{20}$

Non di rado è la morte che si affaccia in queste missive, divenendone quasi inevitabilmente il centro, soprattutto quando a morire sono i figli di qualcuno. Spesso ci si riferisce alla morte con perifrasi eufemistiche (7b) e talora si indugia in lunghe descrizione di riti funebri, non riportate in questa sede per esigenze di sintesi:

7) [a] meglio morire che mal campare [b] Mi dicevi che vostra Madre è passata unaltera vita [c] l'anno scorso aveva uno che giaceva inmezzo alla camera ornato di fiori $[\mathbf{d}]$ si rinnovò di bel nuovo la piaga della mia perdenza rilivendo anche tu ai avuto la perdita di tuo figlio piccino e essendo che e sgravata ad ma però ha fatto una bambina morta essa stava bene ma però stava dispiaciuta

In $7 \mathrm{~d}$ la morte incrocia tragicamente una nascita (o, per meglio dire, una non-nascita). Le tappe della vita (da quelle dei più giovani a quelle degli adulti) dei famigliari, ivi compresi i sacramenti, sono un altro tema ricorrente di lettere che hanno come funzione principale quella di tenere insieme pezzi di famiglia separati da un oceano.

Salute, malattia e morte sono spesso interpretate alla luce di una vigorosa fede nella Divina Provvidenza. La religiosità delle comunità italoamericane $e^{21}$, espressa attraverso la celebrazione, oltre oceano, delle feste patronali italiane (Pretelli 2011: 63-64), innerva le missive, attraversando l'intero secolo in questione. Che si tratti dell'espressione di un'effettiva convinzione o di mera formula epistolare, il richiamo al volere di Dio o a un non meglio precisato destino (8d), cui migranti si piegano rassegnati, è una presenza costante nel testo:

8) [a] Grazie Iddio stiamo tutti e due alquando bene [b] Con sommo dispiacere ti annunzio la morte del tuo fratello $X[. .$.$] . adesso caro cognato non resta altro che la rassegnazione verso la volontà di Dio$ con la morte non ce che fare siamo nati e dobbiamo morire [c] contro la mano divina nessuno può vincere [d] il destino certamente non lo facciamo noi siamo già destinati da Dio [e] Ogni sera mi raccomando i miei perduti e gli dico che preghessero per la salute mia che sono degni di preghiere e prego anche per te che ti faccia star bene e ti ritirasse presto in famiglia per consolazione tua [f] Dio possa lucitarmi la mente di togliermi tante cattivi pensieri che si presentano nelle cervelle

Non mancano, rispetto all'accettazione di un destino avverso, gli accenti polemici, rivolti talora direttamente a Dio, in forma di constatazione (9b) o di vera e propria invettiva (9c):

20 Qui e altrove, per ragioni di riservatezza, mi è stato chiesto di non riportare nomi e cognomi (sostituiti, nel testo, da una $X$ ) delle persone menzionate nelle lettere.

21 Alla religiosità, per lo più cattolica, espressa nei quartieri etnici e sostenuta dalla rete parrocchiale, fa da contraltare la diffusione delle idee socialiste e anarchiche, che, in seno alle comunità italiane, trova espressione in associazioni sindacali e nella stampa radical. Tali idee, ad ogni modo, sono estranee ai testi considerati nel presente contributo. 
9) [a] per me non è Natale perché come sapete quel giorno fu per me il giorno del mal destino [b] Cristo si è voluto ben divertire verso lenostre case ma come si a divertito verso la casa mia sono cose che dovranno andare nelle storie [c] Ah maledetto destino come serrasti gli occhi verso la casa mia [d] io di voi non mi ho mai dimenticato come Cristo si ha dimenticato di me

Si è già accennato alla centralità della famiglia. Quest'ultima è spesso vista come un nucleo di persone di cui va preservata l'armonia e l'allontanamento (causato dall'emigrazione o da altre ragioni) dal quale, rappresenta sempre un motivo di sofferenza (10b, 10c). Le tappe della vita dei famigliari (come si è già visto ai punti 6-8), dalle nascite alle morti, dai battesimi ai matrimoni, fino alle piccole novità quotidiane (10d), sono temi su cui si cercano notizie e su cui si danno notizie:

10) [a] Saluto tutti di casa nostra e voglio che si vive sem pre in buona armonia [b] è già un anno completo che stai lontano dalla famiglia [c] mi auguro che ottenessi una piccola licenza per passare quei giorni inmezzo alla tua famiglia [d] il piccolo X comincia a camminare bene

La ragione principale, quando non l'unica, per intraprendere un progetto migratorio, è ovviamente quella economica. L'attenzione al denaro, al valore e al costo dei beni materiali e dei servizi, così come l'aggiornamento sugli aspetti economici del lavoro è ricorrente in quasi tutte le lettere, che ci forniscono interessanti testimonianze su questi aspetti della società americana (osservata dal punto di vista della popolazione immigrata) ${ }^{22}$. Talora gli scriventi riferiscono di prezzi precisi (11e); talora la valutazione, approssimativa, viene enunciata attraverso espressioni vaghe e metaforiche (11c). In ogni caso, tutto ciò che passa sotto gli occhi del migrante sembra essere valutato sotto l'aspetto economico:

11) [a] Laffari vanno troppo male [b] pure il giorno della S.S. Trinità avete fatto mediocri affari [c] attendo al mio lavoro e la gente invidia a chi si lucra un tozzo di pane basta [d] Quanta anni ci vuole per quadagnare quelle soldi ma noi scriveremo a X se deve moderare il prezzo [e] Xè stato fortunato a tutto comprò una casa che costava al di sopra di 100 mila dollare lui la pagà appena 60 mila e volendola vendere oggi la vende col doppio del prezzo

I riferimenti al denaro sono legati anche a un aspetto pratico: non di rado le lettere accompagnano l'invio di somme di denaro, di cui forniscono un dettagliato resoconto (12a). Una funzione simile, sia dal punto di vista pratico sia dal punto di vista psicologico, è svolta dalle minuziose descrizioni delle merci che accompagnano talune missive, allo scopo di evidenziarne il valore e verificarne il corretto arrivo a destinazione (12b):

12) [a] ci mandai anche 125 lire cioè le 25 lire mandai 5 lire scascuno ai tuoi figli 5 lire a tua moglie e 5 lire gli disse che lavessero mandate per mio complimento [b] Mando una camicetta di seta alla tua figlia $X$ dunque la camicetta e molto bella e credo che la ragazza deve rimane re contenta e per segno vicino alla camicetta alla parte di nanzi da sotto alla piega gli ho fatto un segno col

22 Cfr. le considerazioni di Gibelli - Caffarena (2001: 572-573). 
cotone rosso per motivo che tante volte la dovessero campiare perciò scrivetemi subito se questi oggetti l'hai ricevuti [c] Cara moglie mi hai scritto diverse volte ma non mi hai fatto sapere se hai ricevuto i miei panni che mandai [d] in unaltra mia lettera ti spedirò altri Francobolli cosi scriverai spesso

Come accennato, queste lettere ci forniscono anche informazioni sullo stato della vita materiale nella società italiana e italoamericana. Particolarmente curiosa, a questo proposito, può essere l'allusione ai francobolli di $12 \mathrm{~d}$. In assenza di informazioni circostanziate, si possono formulare almeno due ipotesi: la prima è che, in seno alle Little Italies, fosse attiva una circolazione di francobolli italiani, usati come moneta o, appunto, spediti in patria per ricevere lettere dalla famiglia di origine; la seconda, forse più onerosa ma non necessariamente improbabile, è che negli Stati Uniti venissero falsificati francobolli italiani ${ }^{23}$. Alcune informazioni riguardano il cibo e l'alimentazione, talvolta con riferimento a prodotti che vengono spediti da un capo all'altro dell'oceano $(13 \mathrm{c}, 13 \mathrm{~d}, 13 \mathrm{e}, 13 \mathrm{f}, 13 \mathrm{~g})$. Anche per ragioni economiche ${ }^{24}$, il flusso non è, come si potrebbe essere portati a pensare, a senso unico (dall'Italia all'America). Va dunque circostanziata l'affermazione che "[g]li italiani immigrati disdegnavano il cibo "americano"” (Vecoli 2002: 59) ${ }^{25}$ :

13) [a] tutti i giorni mi compro qualche cosa in cantina, a me la pasta non tanto mi piace e massimo il riso non mi piace affatto. E quindi è necessario che ogni sera mi compro una pietanza di qualunque cosa sia, cioè di carne di uova, di piselli di fagioli e via discorrendo [b] non si può avere zucchero il primo alim ento che ci vuole in America la farina molto cara [c] io qui compro ciò che mi piace per esempio quel formaggio che tu mandasti a me e tuo cognato X io pagai 200 lire $q u i$ [d] come sai ho ricevuto i due cacicavalli [e] Il 27 scorso ricevei il prigiotto giusto come tu mi parlavi e laccettò ansioso come anche io rimase contento però facesti un errore a togliere la coteca da vicino mentre io quella voleva per farmi la minestra [f] per la prossima Pasqua mi devi mandare un po' di ventresca e un po' di salamo ossia supersate $[\mathbf{g}]$ vi ringrazio del pregiotto che mi avevi mandato

Non mancano gli accenni alla realtà contadina irpina, sia in forma di raccomandazioni (14a) sia in forma di resoconto (14b):

14) [a] Ti raccomando quando prima di trovare otto nove donne per andare a defrancere la vigna, in modo che non si fa a palude piena d'erba. / Se potrai entrare nella pagliaia quelle fascine di

23 La falsificazione di francobolli italiani all'estero è un fatto accertato. Un caso noto e ben documentato è quello del cosiddetto "falso di Buenos Aires": nei primi anni ‘50, un falsario imitò francobolli italiani nella capitale argentina. Questi francobolli venivano poi inviati in patria e usati per spedire lettere e pacchi verso il continente sudamericano (Carraro Carraro 2012: 34). Debbo questa informazione alla cortesia e alla competenza filatelica di Giacomo Giuntoli.

24 L'aspetto economico riguarda, ovviamente, anche le considerazioni sul cibo, bene di consumo fra gli altri: cfr. 13b e 13c.

25 Uno dei prodotti (fra quelli menzionati nelle lettere) che venivano spediti più spesso dal nuovo mondo al vecchio è il caffè: Il fidanzato di mia figlia $X$ ha spedito un pò di caffé; io fra giorni vi faccio un pacco di caffe; timanto due scatoli di caffe. Inutile sottolineare il valore, anche simbolico, del caffè per un italiano, in particolar modo per un campano. Curiosamente, il caffè è frequentemente evocato, come immagine, anche in formule autoaugurali: il resto vi fati una tazza di caffe per mio ricordo; vi fati una tazza di caffe per nostro ricordo; te fate una tazza di caffe per amore mio. 
salmenti farai molto bene, in modo che non se la prendono quando viene la mietitura, come già abbiamo ricapitato diversi anni $[\mathbf{b}]$ inquando ai due maiali che ti morirono non darti nessun pensiero perchjè gli animali si possono sempre comperare d'ovunque e la carne umana no

"Nell'entrare in contatto con un'economia industriale fortemente sviluppata, pochi [emigranti] avevano esperienza di occupazioni non legate all'agricoltura": se quest'affermazione di Rudolph J. Vecoli (2002: 57) può essere considerata valida, al netto delle dovute eccezioni, per l'emigrazione italiana negli USA nel suo complesso, ciò è tanto più vero per gli immigrati di origine meridionale. Lo spostamento non è solo geografico, ma anche socioeconomico: da una realtà rurale e contadina si passa a una realtà urbana e industrializzata. Queste differenze non mancano di colpire i migranti e offrono loro lo spunto per parlare, nelle missive, dell'America stessa. Si istituisce un confronto, implicito o esplicito, che a volte unisce, a volte divide i due paesi. Non di rado a vincere la competizione, se cosi si può dire, è proprio l'Italia (15e, 15f), il luogo degli affetti più cari, della genuinità e di una memoria idealizzata ${ }^{26}$ :

15) [a] va bene che è in America ma caro fratello dallora della guerra qui è peggio ancora dell'Italia $i$ veveri cari la gente non lucra per quanto si spende anche la suola la pagammo 10 soldi loncia [b] anche l'America si comincia ad odorare la mala vita perché sta anche in Guerra per aiuto degli Alleati e non si può comprare niente [c] lamerica è mala combinata con un piccolo tantino può finire tutto [d] Scranton è una città abbastanza grande [e] qui c’è puro la manna celeste, tanto ti ho detto poi la ventresca che mi hai forzato e qui ci stanno ma non tengono quel sapore [f] la sorella $X$ sta peggio di me e nessuno può credere di questa maledetta america i figli in che modo si istruiscono non possono trovare niente da fare

La lettura complessiva delle missive, scritte lungo l'arco di un secolo, permette di rifiutare le teorie assimilazioniste di Handlin (1951), secondo cui la cultura etnica delle comunità immigrate, tanto più quelle dell'Europa del Sud, avrebbe ceduto presto e senza opporre resistenze alla pressione della cultura americana. Il fenomeno, in realtà, è molto più complesso: non solo perché, talora, l'emigrazione era vista come qualcosa di temporaneo (Vecoli 2002: 56-57), né soltanto per i rigurgiti xenofobi di cui gli italiani furono vittime (Stella - Franzina 2001); ma anche per il forte legame che gli emigranti, non solo quelli di prima generazione, hanno sempre mantenuto con i parenti rimasti in patria. Non di rado sono le generazioni successive, meno italofone ma spesso più scolarizzate, a prendere la penna per scrivere ai propri parenti italiani: colpisce, in questo caso, oltre alla continuità di rapporti, quella culturale, che non esibisce fratture (quanto a tematiche e atteggiamenti) fra prima generazione e successive.

Il rapporto fra società americana e comunità etnica, dunque, comporta una negoziazione: ciò si compie, nel caso di Stati Uniti (e Canada) e Italia, soprattutto nelle Little Italies, terreno

26 Lepistolografia italoamericana fornisce anche esempi di segno opposto: come ricorda Pretelli (2011: 41), sull'incremento dei flussi migratori dall'Italia agli States di inizio Novecento "non influì solo la rete degli imbonitori, ma anche i racconti, le testimonianze e le lettere degli emigrati che contribuirono in maniera consistente alla promozione del mito dell'America". Cfr. anche Gibelli - Caffarena (2001: 572): i due autori ricordano come questo tipo di documenti, non di rado, fosse pubblicato sulla stampa per alimentare o per frenare (a seconda del tono della lettera in questione) un dato flusso migratorio. 
di coltura delle enclaves italoamericane. La presenza di gruppi di italiani, naturalmente, non sfugge all'attenzione degli emigranti (16a) ed entra nei loro resoconti, non privi di giudizi di valore (16b), dovuti anche al carattere regionale di tali comunità, in cui spesso si riproducevano dinamiche sociali (campanilismo) simili a quelle attive nella Penisola ${ }^{27}$ :

16) [a] piccoli paesi intorno a Scranton dove ci stanno assai italiani [b] è buono di brogliare con i taliani stupidi

L'epistolografia migrante non può sottrarsi al racconto del viaggio che, se compiuto in nave, assume i contorni dell'esperienza epica, contribuendo a fondare dei veri e propri topoi narrativi, riscontrabili in modo incredibilmente simile in diversi epistolari ${ }^{28}$ :

17) Il mare sempre in tempesta fino ad ora, non si mangia e ne si dorme tante cose che si perdono ma nesiuno mangia, tutti che rovesiano li vedi chi a una parte e chi a unaltra tutti mezzi morti per terra, se ci vedete come abbiamo fatto ancora con due giorni e mezzo di viaggio, mimaggino finché si arriva a destinazione spero di arrivare presto, se no proprio mi sfastidio non tanto per me ma per $X$, che rovescia pure

Con questa breve rassegna, non esaustiva, si è voluto dare un'idea dell'interesse che i contenuti di queste lettere possono suscitare nel lettore moderno. Talora, al netto delle incertezze linguistiche, si tratta di documenti che possono essere letti come delle vere e proprie novelle, che alternano momenti narrativi a slanci lirici, con echi letterari (cfr., per es., 9c) di cui sarebbe interessante stabilire la provenienza.

Fortissima è la tensione emotiva di tali testi, la cui stesura rappresenta uno sforzo enorme da parte di scriventi semicolti ${ }^{29}$, coscienti della propria condizione socioculturale. Lo sforzo compiuto per scrivere è un altro tema, costante negli epistolari migranti, che non manca neppure in questo: Perdonerai il male scritto, scrive uno dei mittenti al proprio destinatario. L'insicurezza psicologica di fronte alla pagina bianca condiziona, dunque, sia la forma (come testimoniano gli ipercorrettismi, cui si è fatto accenno in 2e), sia il contenuto dei testi, nonché i tempi di stesura e, di conseguenza, i ritmi dello scambio comunicativo ${ }^{30}$.

L'insicurezza scrittoria di persone che sono, con ogni evidenza, più avvezze a servirsi della lingua parlata, produce evidenti interferenze diamesiche: alcuni dei tratti linguistici esemplificati

27 Sulle identità regionali delle Little Italies cfr. Harney - Scarpaci (1981) e Garroni (2002). Attorno ai quartieri etnici si addensano, naturalmente, consolidati stereotipi. L'inadeguatezza dei canoni descrittivi tradizionali è sentita in De Biase (2012). Portelli (2000, 2004) si serve di fonti diversificate (letterarie e non) per affrontare e, talora, superare i modelli tradizionali etnici.

28 Cfr., a questo proposito, la lettera datata 14.2.1926 riportata in Gibelli - Caffarena (2001: 570), in particolare, il brano ripreso in Bianco (2013: 104).

29 Sull'italiano dei semicolti e sull'italiano popolare (etichetta quasi sovrapponibile, se non nella teoria, almeno nella pratica) cfr. Cortelazzo (1972) e D’Achille (1994, 2010).

30 A questo proposito, cfr. l'estratto dalla lettera di un emigrante italiano in Argentina riportata da Gibelli e Caffarena: "Non ho potuto scriverti prima perchè sicome io non so scrivere; non pozio faro a volontà" (2001: 564). 
ai punti 1-5, oltre al generale modo di costruire il discorso (ridondante, privo di coerenza tematica, ricco di frasi esclamative, etc.), sono segni di una scrittura che "tende naturalmente ad avvicinarsi all'oralità" (Gibelli - Caffarena 2001: 565), talora assumendo le forme di un discorso teatralizzato.

Gli emigranti intingono il pennino nel calamaio spinti dalla necessità di mantenere rapporti che altrimenti si sfilaccerebbero; lo fanno, tuttavia, quasi sempre sotto l'impulso di una ragione particolare, di un'occasione speciale: una nascita, una morte, un avvenimento importante, l'invio di beni materiali, ecc. Ciò non impedisce a queste lettere di assorbire anche stralci di semplice vita quotidiana, che rappresentano con vivacità e spesso con precisione.

Una pubblicazione integrale dei testi e un'analisi sistematica delle storie ivi raccontate getterà sicuramente una luce su alcuni aspetti dell'emigrazione italiana all'estero e, nello specifico, dell'emigrazione irpina in Nord America: un'esperienza umana, individuale e collettiva, che ha cambiato la storia italiana e americana, stabilendo un ponte fra centro e periferia "e facendo sentire tutto il peso dei processi migratori nei fattori di crescita e crisi della società urbana" (Pitto 2005); una storia fatta di successi, che ben conosciamo, ma anche di insuccessi, spesso trascurati dalla memoria; una storia che ha ispirato scrittori e registi, che esercita un fascino per il coraggio con cui i protagonisti di questi viaggi epici, di queste avventure pluridecennali hanno affrontato il distacco, i pregiudizi, la fame, le difficoltà materiali e psicologiche del progetto migratorio ${ }^{31}$. Come si legge, scritto in modo lapidario, in una lettera del 1926: "L'Emigrazione per me è stato un castigo".

\section{Riferimenti bibliografici}

Bevilacqua, P., De Clementi, A., \& Franzina, E. (Eds.). (2001-2002). Storia dellemigrazione italiana. 2 voll. Roma: Donzelli.

Bianco, F. (2004). La coniunctio relativa nell'italiano antico. In: M. Dardano, \& G. Frenguelli (Eds.), SintAnt. La sintassi dellitaliano antico [online] (pp. 485-488). Roma: Aracne. In: http://www.francescobianco.net/linguistica/coniunctio/.

- (2009). L'aggettivo relativo in italiano antico. Bollettino dell'Atlante Lessicale degli Antichi Volgari Italiani [online], 2, 31-54. In: http://www.francescobianco.net/linguistica/balavi2009/.

. (2012). L'aggettivo relativo fra italiano antico e moderno. In: P. Bianchi et al. (Eds.), La variazione

nell'italiano e nella sua storia. Varietà e varianti linguistiche e testuali. Atti dellXI Congresso SILFI Società Internazionale di Linguistica e Filologia Italiana (Napoli, 5-7 ottobre 2010) [online] (vol. 1) (pp. 309-318). Firenze: Cesati. In: http://www.francescobianco.net/linguistica/silfi2010/.

(2013). Le lettere dei migranti irpini fra italiano, dialetto e lingua straniera. In: Cristina Albizu et al. (Eds.), Variante et varieté - Variante e varietà - Variante y variedad - Variante und Varietät. Actes du VI Dies Romanicus Turicensis, Zurich, 24-25 juin 2011 [online] (pp. 101-117). Pisa: ETS. In: http://www. francescobianco.net/linguistica/zurigo2011/.

31 La storia dell'emigrazione italiana negli USA, considerata attraverso i suoi riflessi letterari, è oggetto dei volumi di Marazzi (2011) e, relativamente ad alcuni capitoli, di Portelli (2004). 
. (2016). Burocratese nascosto nell'italiano moderno. In: G. Ruffino, \& M. Castiglione (Eds.), La lingua variabile nei testi letterari, artistici e funzionali contemporanei. Analisi, interpretazione, traduzione. Atti del XIII Congresso SILFI Società Internazionale di linguistica e Filologia Italiana, Palermo 22-24 settembre 2014 [online] (pp. 519-528). Firenze: Cesati. In: http://www.francescobianco.net/linguistica/silfi2014/.

Carraro, D., \& Carraro, G. (2012). Catalogo delle specializzazioni e varietà della Repubblica Italiana e Trieste. Roma: Sassone.

Cortelazzo, M. (1972). Avviamento critico allo studio della dialettologia italiana. $3^{\circ}$ vol. Lineamenti di italiano popolare. Pisa: Pacini.

D’Achille, P. (1994). L'italiano dei semicolti. In: L. Serianni, \& P. Trifone (Eds.), Storia della lingua italiana (vol. 2). Scritto e parlato (41-79). Torino: Einaudi.

- (2010). Italiano popolare. In: R. Simone (Ed.), Enciclopedia dellitaliano [online] (vol. 1) (pp. 723-726). Roma: Istituto della Enciclopedia Italiana. In: http://www.treccani.it/enciclopedia/italianopopolare_(Enciclopedia_dell'Italiano)/.

De Biase, M. (2012). Infami senza lode. Etnografia dei migranti italiani a Toronto e dei "rimasti" in Italia. Verona: Ombre corte.

Garroni, M. S. (2001-2002). Little Italies. In: P. Bevilacqua, A. De Clementi, \& E. Franzina (Eds.), Storia dellemigrazione italiana (vol. 2) (pp. 207-233). Roma: Donzelli.

Gibelli, A., \& Caffarena, F. (2001-2002). Le lettere degli emigranti. In: P. Bevilacqua, A. De Clementi, \& E. Franzina (Eds.), Storia dellemigrazione italiana (vol. 1) (pp. 563-574) Roma: Donzelli.

Handlin, O. (1951). The Uprooted. Boston: Grossett \& Dunlap.

Harney, R. F., \& Scarpaci, V. J. (1981). Little Italies in North America. Toronto: The Multicultural Society of Ontario.

Lagumina, S. J. et al. (Eds.). (2000). The Italian American Experience. An Encyclopedia. New York: Garland. Longo, S. (2004). Italians of Northeastern Pennsylvania. Charleston: Arcadia Publishing.

Marazzi, M. (2011). A occhi aperti. Letteratura dellemigrazione e mito americano. Milano: FrancoAngeli.

Pitto, C. (2005). La città come stile di vita nell'identità migrante. In: P. Palmeri (Ed.), I rapporti interculturali in Italia oggi. Una prospettiva antropologica [online] (pp. 197-249). Padova: CLEUP. In: http://bottegantropos.altervista.org/rel_palmieri.htm [si cita dalla versione online].

Portelli, A. (2001-2002). L’immaginario e le rappresentazioni. Nella letteratura di lingua inglese. In: P. Bevilacqua, A. De Clementi, \& E. Franzina (Eds.), Storia dellemigrazione italiana (vol. 2) (pp.613-631). Roma: Donzelli.

Pretelli, M. (2011). L'emigrazione italiana negli Stati Uniti. Bologna: Il Mulino.

Stella, G. A., Franzina, E. (2001-2002). Brutta gente. Il razzismo anti-italiano. In: P. Bevilacqua, A. De Clementi, \& E. Franzina (Eds.), Storia dellemigrazione italiana (vol. 2) (pp. 283-311). Roma: Donzelli.

Vecoli, R. J. (2001-2002). Negli Stati Uniti. In: P. Bevilacqua, A. De Clementi, \& E. Franzina (Eds.), Storia dellemigrazione italiana (vol. 2) (pp. 55-88). Roma: Donzelli. In: http://win.ecoistitutoticino.org/emigrazione/vecoli-web.htm.

Vedovelli, M. (Ed.). (2011). Storia linguistica dellemigrazione italiana nel mondo. Roma: Carocci. 
